\title{
Comunicación
}

\section{Frecuencia de Neoplasias en Cavidad Oral de Caninos y Felinos, Diagnosticadas Histopatológicamente en el Laboratorio de Pato- logía Veterinaria de la Universidad Nacional Mayor de San Marcos (Periodo 2007- 2013)}

\author{
Frequency of Oral Cavity Neoplams in Dogs and Cats, Histopathologically \\ Diagnosed in the Veterinary Pathology Laboratory of the National University \\ of San Marcos (Period 2007-2013) \\ Jhessenia Páucar P. ${ }^{1}$, Rosa Perales C.,2, Luis Tabacchi N. ${ }^{1}$
}

\section{Resumen}

El objetivo del presente estudio retrospectivo fue determinar la frecuencia de neoplasias en cavidad oral en caninos y felinos mediante el diagnóstico histopatológico en el Laboratorio de Patología Veterinaria de la Universidad Nacional Mayor de San Marcos, Lima, Perú, durante el periodo 2007-2013. Se diagnosticaron 115 casos de neoplasias orales dentro de 1454 casos de neoplasias caninas $(7.9 \%)$ y 7 casos de neoplasias orales dentro de 54 casos de neoplasias felinas (13\%). El épuli fue la neoplasia de mayor frecuencia $(20.5 \%)$.

Palabras clave: cavidad oral, neoplasia, frecuencia, caninos, felinos

\section{AbSTraCt}

The aim of this retrospective study was to determine the frequency of neoplasms in the oral cavity in canines and felines that were histopathologically diagnosed in the Veterinary Pathology Laboratory of the National University of San Marcos, Lima, Peru, in the period 2007 to 2013 . A total of 115 cases of oral tumors were diagnosed in 1454 cases of canine tumors $(7.9 \%)$ and 7 cases of oral tumors in 54 cases of feline tumors $(13 \%)$. The epuli was the most frequent neoplasia $(20.5 \%)$.

Key words: oral cavity, neoplasm, frequency, canines, felines

${ }^{1}$ Laboratorio de Histología, Embriología y Patología Veterinaria, Facultad de Medicina Veterinaria, Universidad Nacional Mayor de San Marcos, Lima, Perú

${ }^{2}$ E-mail: rperales_fmv@hotmail.com

Recibido: 5 de febrero de 2016

Aceptado para publicación: 15 de mayo de 2016 


\section{INTRODUCCIÓN}

El diagnóstico de patologías tumorales en caninos y felinos ha aumentado en forma progresiva en las últimas décadas, llegando las neoplasias a situarse como una de las principales causas de muerte en animales adultos y viejos (Mialot y Lagadic, 1990).

Las neoplasias orales son consideradas el cuarto tipo de mayor frecuencia en caninos y felinos, y representan el $6 \%$ de los tumores caninos y cerca del 3\% de los tumores felinos (Foale y Demetriou, 2011). En el Perú se han realizado diversos estudios sobre neoplasias en mascotas, especialmente a base de la casuística (Chau et al., 2010; Castro et al., 2010; Vicente et al., 2015); sin embargo, neoplasias en la cavidad oral han sido poco estudiadas.

El presente estudio tuvo como objetivo determinar la frecuencia de neoplasias en la cavidad oral en caninos y felinos, tomando como fuente de información los diagnósticos histopatológicos del laboratorio de patología veterinaria de la Universidad Nacional Mayor de San Marcos, en el periodo de 2007 a 2013, a fin de contribuir al conocimiento del comportamiento biológico de las neoplasias.

\section{Materiales y Métodos}

Se realizó un estudio retrospectivo con base en los informes histopatológicos de neoplasias caninas y felinas de la cavidad oral efectuados en el Laboratorio de Patología de la Facultad de Medicina Veterinaria de la Universidad Nacional Mayor de San Marcos, Lima, Perú, durante el periodo de enero de 2007 a diciembre de 2013.

Se consideraron las siguientes variables: raza (pura y cruzada), edad ( $0 \mathrm{a}<1,1 \mathrm{a}<6,6$ $\mathrm{a}<11, \geq 11$ años en caninos y $0 \mathrm{a}<5,5 \mathrm{a}<9$, $\geq 9$ años en felinos), sexo, localización ana- tómica (labio, encía, paladar, lengua, tonsila) y diagnóstico histopatológico. Se determinó la frecuencia de neoplasias ( \pm intervalo de confianza al 95\%) de la cavidad oral con relación al total de neoplasias en caninos y felinos.

\section{Resultados}

El laboratorio tuvo un registro de 1508 neoplasias en caninos y felinos, de los cuales 122 fueron diagnosticadas como neoplasias en la cavidad oral. En caninos se tuvo una frecuencia de $7.9 \pm 1.3 \%(115 / 1454)$ y en felinos de 13.0 $\pm 8.9 \%$ (7/54) (Cuadro 1). En los caninos, las razas con mayor presentación de neoplasias fueron el Bóxer (11.0\%), Labrador Retriever (9.2\%), Rottweiller (7.3\%) y Pastor Alemán (5.5\%), mientras que en los felinos solo se presentaron en mestizos.

Las neoplasias encontradas en la cavidad oral, según el diagnóstico histopatológico, se muestran en el Cuadro 2. El épuli fue el tipo de neoplasia más común (20.5\%), seguido del rabdomiosarcoma (17.2\%; Figura 1) y el carcinoma de células escamosas $(13.9 \%$, Figura 2).

\section{Discusión}

El 7.9\% de frecuencia de neoplasias en cavidad oral en caninos fue similar a los reportes de Lucena et al. (1996), Venturini (2006) y Liptak y Withrow (2012), quienes reportaron frecuencias de $5.6,5.0$ y $7.0 \%$ de casos, respectivamente. En el caso de felinos, el $13 \%$ fue superior al $3 \%$ encontrado por Liptak y Withrow (2012).

En ambas especies se observó una mayor frecuencia de presentación en machos que en hembras, lo cual coincide con otros reportes (Howard, 2000; Gioso, 2003; Mendiz, 2004); sin embargo, no se han encontrado estudios que plantearan asociaciones entre 
Cuadro 1. Distribución de casos de neoplasias en cavidad oral en caninos y felinos, según registros del Laboratorio de Patología de la Universidad Nacional Mayor de San Marcos (2007-2013)

\begin{tabular}{llcc}
\hline Variable & & $\begin{array}{c}\text { Casos } \\
(\mathrm{n})\end{array}$ & $\begin{array}{c}\text { Frecuencia } \\
(\%)\end{array}$ \\
\hline Sexo & Macho & 47 & 61.0 \\
& Hembra & 72 & 39.0 \\
Edad (caninos) & $0 \mathrm{a}<1$ & 2 & 1.8 \\
& $1 \mathrm{a}<6$ & 22 & 19.6 \\
& $6 \mathrm{a}<11$ & 55 & 49.1 \\
Edad (felinos) & $=11$ & 33 & 29.5 \\
& 0 a $<5$ & 4 & 57.1 \\
Raza (caninos) & $5 \mathrm{a}<9$ & 1 & 14.3 \\
& $=9$ & 2 & 28.6 \\
Localización & Pura & 82 & 75.2 \\
& Mestizo & 27 & 24.8 \\
& Gingiva & 69 & 57.0 \\
& Labio & 26 & 21.0 \\
& Paladar & 9 & 7.0 \\
& Lengua & 9 & 7.0 \\
& Otros & 8 & 7.0 \\
& Tonsila & 1 & 1 \\
\hline
\end{tabular}

este tipo de neoplasias e influencias hormonales.

La mayor frecuencia de presentación de estas neoplasias orales en caninos fue en animales mayores de 6 años, debido a una mayor predisposición a desarrollar este tipo de tumores por la activación de oncogenes y probables elementos cancerígenos (Foale y Demetriou, 2011). Por otro lado, en los felinos se encontró una mayor frecuencia en animales menores de 5 años, difiriendo con otros autores que reportan una mayor presentación de neoplasias entre los 8 y 14 años de edad (Gloaguen, 2009; Castro et al., 2012).
Si bien se encontraron ciertas diferencias entre razas de caninos, la muestra no fue lo suficientemente grande para permitir sacar conclusiones de tipo genético. En este sentido, Lucena et al. (1996) atribuyeron la predisposición racial a razones geográficas.

La gingiva como principal zona afectada por las neoplasias (57\%) coincide con lo descrito por Gioso (2003) y Mendiz (2004).

Los tumores malignos tuvieron mayor frecuencia $(72 \%)$ en comparación con los benignos. Asimismo, la mayor frecuencia de rabdomiosarcoma en caninos difiere con los 
Cuadro 2. Neoplasias orales en caninos y felinos según diagnóstico histopatológico del Laboratorio de Patología Veterinaria, Universidad Nacional Mayor de San Marcos, Lima (2007-2013)

\begin{tabular}{lcc}
\hline Diagnóstico & Casos & $\begin{array}{c}\text { Frecuencia } \\
(\%)\end{array}$ \\
\hline Épuli & $(\mathrm{n})$ & 20.5 \\
Rabdomiosarcoma & 25 & 17.2 \\
Carcinoma de células escamosas & 21 & 13.9 \\
Melanoma & 17 & 10.7 \\
Plasmocitoma & 13 & 9.8 \\
Hemangiosarcoma & 12 & 6.6 \\
Papiloma & 8 & 5.7 \\
Adenocarcinoma de células basales y glándula sebácea & 7 & 4.9 \\
Fibrosarcoma & 6 & 4.1 \\
Linfoma maligno & 5 & 2.5 \\
Mastocitoma & 3 & 1.6 \\
Tumor venéreo transmisible extragenital & 2 & 1.6 \\
Osteosarcoma & 2 & 0.8 \\
\hline
\end{tabular}

reportes de Lucena et al. (1996) y Roza et al. (2009), quienes indican que el carcinoma de células escamosas fue la neoplasia maligna más común, seguida del fibrosarcoma y el melanoma. En la especie felina, donde solo se encontraron neoplasias malignas, siendo el carcinoma de células escamosas la más frecuente (43\%), coincidió con el estudio de Stebbins et al. (1989).

\section{Conclusiones}

La frecuencia de neoplasias en cavidad oral encontradas en el Laboratorio de Patología de la Universidad Nacional Mayor de San Marcos en el periodo 2007-2013 fue de $7.9 \%$ en caninos y de $13.0 \%$ en felinos.

\section{Literatura Citada}

1. Castro T, Chavera A, Perales $R$, Fernández V. 2012. Frecuencia de neoplasias en felinos en la ciudad de Lima durante el periodo 1996-2007. Rev. Inv Vet Perú 23: 529-532. doi: 10.15381/ rivep.v23i4.975

2. Chau G, Chavera A, Perales $R$, Gavidia C. 2013. Frecuencia de neoplasias en glándula mamaria de caninos: estudio retrospectivo en el periodo 1992-2006 en la ciudad de Lima, Perú. Rev Inv Vet Perú 24: 72-77. doi: 10.15381/rivep.v24i1.1663

3. Foale R, Demetriou J. 2011. Oncología de pequeños animales. Barcelona: Elsevier. 224 p. 


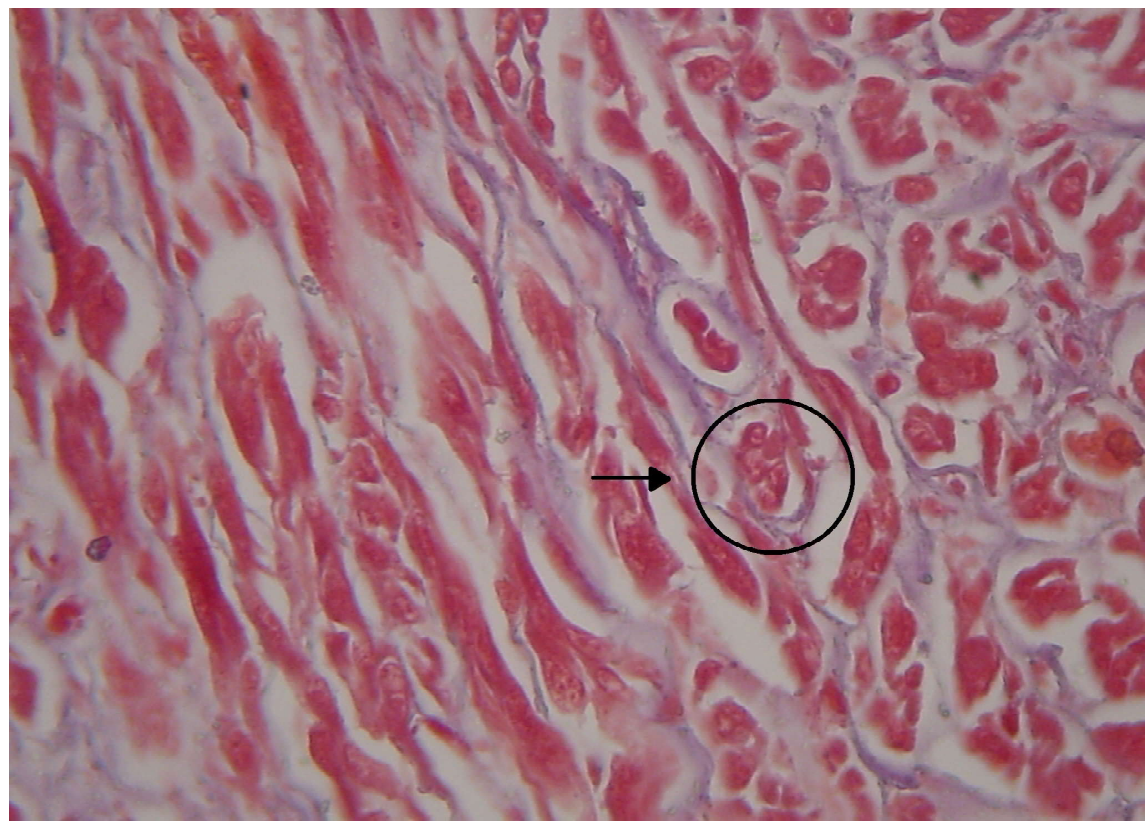

Figura 1. Microfotografía de un rabdomiosarcoma en lengua. Se observan células con varios núcleos $(\rightarrow)(\mathrm{X} 400$, tricrómico de Masson)

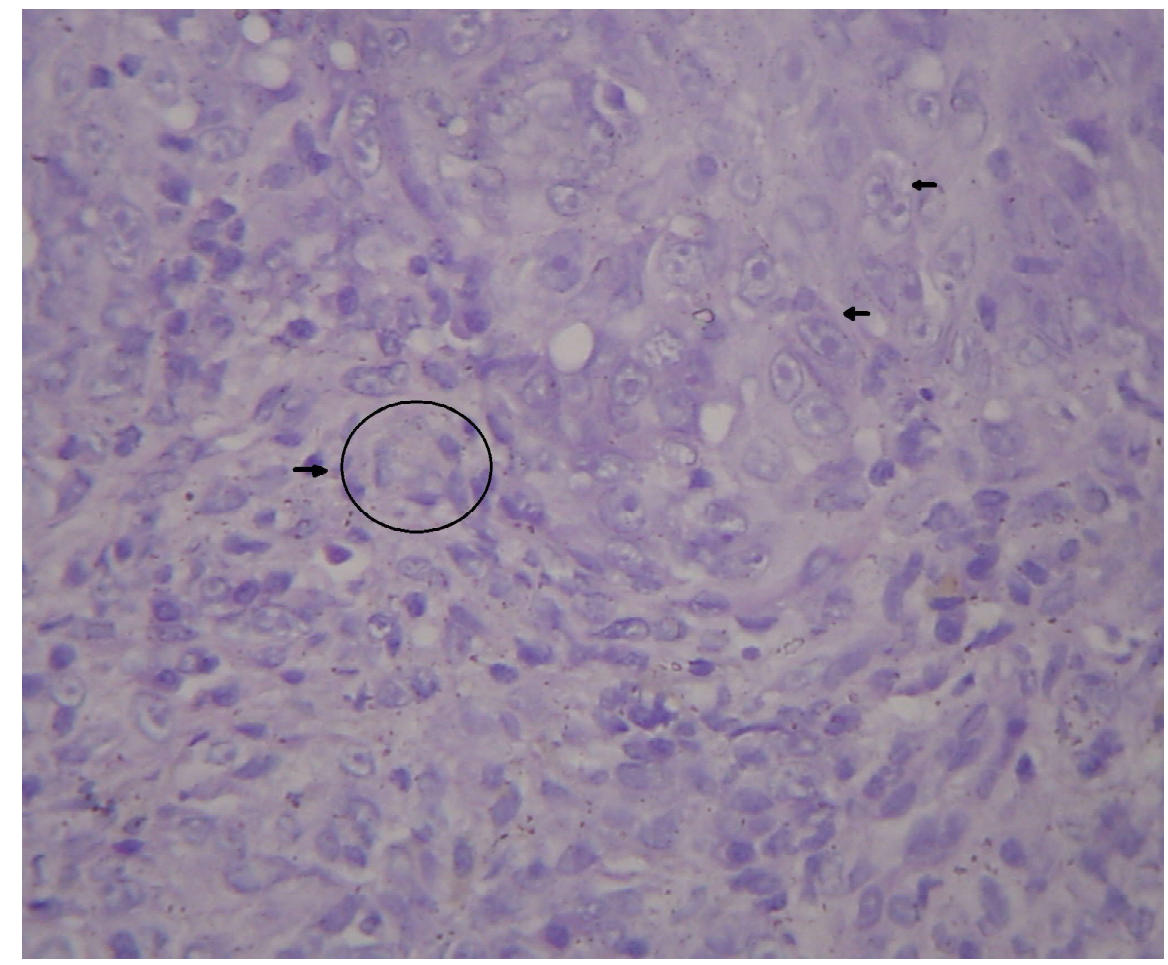

Figura 2. Microfotografía de un carcinoma de células escamosas de la encía de un canino. Se observa la proliferación de células escamosas voluminosas, con nucléolos prominentes $(\rightarrow)$, algunas con dos núcleos que se infiltran a la submucosa (X400, H-E) 
4. Gioso MA. 2003. Neoplasia de cavidade oral. En: Odontología para o clinico de pequenos animais. $5^{\circ} \mathrm{ed}$. Sao Paulo: Editora. p 133-152.

5. Gloaguen T. 2009. Epidemiologie descriptive des tumeurs dans l'espece feline: étude retrospective dans un laboratoire d'anatomie pathologique vétérinaire (2000-2007). These de Docteur Vétérinaire. Nantes: École nationale veterinarie de Nantes. 204 p.

6. Howard P. 2000. Neoplasm of the maxilla and mandible. In: Birchard SJ, Sherding RG (eds). Saunders manual of small animal practice. $2^{\text {nd }}$ ed. Philadelphia: Saunders. p 1076-1078.

7. Liptak JM, Withrow SJ. 2012. Oral tumors. In: Withrow SJ, Vail DM, Page RL (eds). Small animal clinical oncology. $5^{\text {th }}$ ed. Missouri: Elsevier Saunders. $p$ 381-398.

8. Lucena R, Ginel PJ, Pérez J, López R, Chacón F, Mozos E. 1996. Tumores de cavidad oral en el perro: estudio retrospectivo. AVEPA 16: 71-76.

9. Mendiz D. 2004. Descripción clínica y epidemiológica de neoplasias orales en ca ninos y felinos. Tesis de Médico Veterinario. Santiago: Universidad de Chile. 53 p.
10. Mialot M, Lagadic M. 1990. Epidémiologie descriptive des tumeurs du chien et du chat. Rec Med Vet 166: 937 947.

11. Roza MR, Andrade MJ, Almeida DC, Silva LAF, Prieto LA, Souza LAC, Pucci RLQ. 2009. Prevalence of oral neoplasia in dogs diagnosed in 2008. In: $34^{\text {th }}$ World Small Animal Veterinary Congress. São Paulo: WSAVA.

12. Stebbins KE, Morse CC, Goldschmidt MH. 1989. Feline oral neoplasia: a tenyear survey. Vet Pathol 26: 121-128.

13. Venturini M. 2006. Estudo retrospectivo de 3055 animais atendidos no Odontovet (Centro Odontológico Veterinário) durante 44 meses. Tesis de Maestría. São Paulo: Universidade de São Paulo. $104 \mathrm{p}$.

14. Vicente $K$, Perales $R$, Tabacchi L. 2015. Frecuencia histopatológica de neoplasias perianales en caninos: casuística del Laboratorio de Patología Veterinaria de la Universidad Nacional Mayor de San Marcos (2005-2012). Rev Inv Vet Perú 24: 111-117. doi: 10.15381/ rivep.v24i1.1674 